07

\title{
Энергетические характеристики и структура углеродных нанорулонов
}

\author{
(С) А.И. Сягло ${ }^{1}$, А.М. Попов ${ }^{2}$, Н.А. Поклонский ${ }^{1, \uparrow}$ Ю.Е. Лозовик ${ }^{2,3}$ \\ ${ }^{1}$ Белорусский государственный университет, Минск, Беларусь \\ ${ }^{2}$ Институт спектроскопии РАН, Троицк, Москва, Россия \\ ${ }^{3}$ Московский физико-технический институт (Государственный \\ университет), Долгопрудный, Россия \\ ฯE-mail: poklonski@bsu.by
}

Поступило в Редакцию 30 января 2017 г.

С помощью полуаналитических и численных расчетов проведено исследование структурных и энергетических характеристик углеродных нанорулонов, полученных из прямоугольной полоски графена. Определены геометрические размеры полоски графена, из которых возможно получение устойчивых и энергетически выгодных нанорулонов. Рассчитаны зависимости барьеров для сворачивания полоски графена в нанорулон и для разворачивания нанорулона, а также времени жизни нанорулона от размеров полоски. Показана устойчивость при комнатной температуре нанорулонов с длиной перекрытия слоев порядка $1 \mathrm{~nm}$.

DOI: $10.21883 /$ PJTF.2017.14.44830.16721

Открытие [1] и прогресс в развитии методов получения [2-5] углеродных нанорулонов вызвали большой интерес к их электронным [6], оптическим [6] и механическим [7-10] свойствам, а также возможным применениям этих новых углеродных наноструктур. В отличие от многослойных углеродных нанотрубок, состоящих из замкнутых цилиндрических слоев, углеродный цилиндрический нанорулон свернут из единого слоя графена и имеет внутренний и внешний край (см. схему на рис. $1, a)$. Рекордные значения удельной емкости материала на основе углеродных нанорулонов показывают перспективность применения таких материалов в суперконденсаторах [5,11]. Предсказано, что углеродные нанорулоны являются перспективным материалом для хранения водорода [12-14] и использования в газовых сенсорах [15]. Ряд работ посвящен исследованию динамики сворачивания [7] и разворачивания нанорулонов [10], что открывает перспективы для исполь- 
зования нанорулонов в наноэлектромеханических системах. Открытие графена стимулировало получение большого числа других двумерных материалов и гетероструктур, состоящих из слоев разных двумерных материалов. Мы полагаем, что в ближайшем будущем будут разработаны методы получения нанорулонов из других двумерных материалов и гетероструктур на их основе.

Для реализации применений углеродных нанорулонов и разработки методов получения нанорулонов из других двумерных материалов и гетеростуктур необходимо исследование структуры и энергетических характеристик нанорулонов. Для таких исследований были использованы два подхода. Первый подход основан на использовании эмпирических межатомных потенциалов и включает моделирование методом молекулярной динамики $[7,8,10]$ и цепочечную модель, в которой ряд атомов, параллельный оси нанорулона, рассматривается как одна частица [9]. Хотя такой подход позволяет исследовать энергетические характеристики и структуру углеродных нанорулонов, распространение этого подхода на нанорулоны из других материалов требует трудоемкой работы по разработке адекватных межатомных потенциалов. Кроме того, атомистическое моделирование работы наноэлектромеханических систем на основе нанорулонов позволяет рассмотреть только несколько примеров такой системы, а для детального исследования рабочих характеристик системы как функции размеров ее компонент необходимо использовать макроскопические полуаналитические и численные подходы, в которых используются макроскопические выражения для упругой энергии слоя и энергии взаимодействия слоев. В рамках такого подхода было получено приближенное выражение энергии для нанорулона с большим числом слоев [7,8]. Однако наибольший интерес представляют нанорулоны с минимальным числом слоев, что позволяет, например, улучшить адсорбционные свойства материала из нанорулонов или уменьшить размеры наноэлектронных приборов и наноэлектромеханических систем на основе нанорулонов. В отличие от работ [7,8] мы приводим точное выражение для энергии нанорулона, которое применимо для рассмотрения стабильности нанорулонов с минимальным числом слоев и расчета барьеров для сворачивания и разворачивания, а также времени жизни таких нанорулонов из полоски графена. Также нами рассчитаны зависимости структурных характеристик нанорулонов от размеров исходной полоски графена, что позволяет определить минимальные размеры устойчивого нанорулона.

Письма в ЖТФ, 2017, том 43, вып. 14 
Аналогично работам $[7,8]$ полагаем, что расстояние $h$ между соседними слоями нанорулона постоянно и равно расстоянию между слоями графита $0.334 \mathrm{~nm}$. В этом случае расстояние $R$ от слоя нанорулона до его оси описывается в полярных координатах $R$ и $\varphi$ уравнением архимедовой спирали $R=h \varphi / 2 \pi$. Тогда длина $L$ вдоль линии спирали полоски графена, из которой получен нанорулон, связана с внутренним $R_{\text {in }}$ и внешним $R_{\text {out }}$ радиусами нанорулона (см. схему на рис. $\left.1, a\right)$ следующим выражением [16]:

$$
\begin{aligned}
L & =L\left(\varphi_{\text {in }}, \varphi_{\text {out }}\right)=\int_{\varphi_{\text {in }}}^{\varphi_{\text {out }}} \frac{h}{2 \pi} \sqrt{1+\varphi^{2}} d \varphi \\
& =\frac{h}{4 \pi}\left(\varphi_{\text {out }} \sqrt{1+\varphi_{\text {out }}^{2}}-\varphi_{\text {in }} \sqrt{1+\varphi_{\text {in }}^{2}}+\operatorname{arsinh}\left(\varphi_{\text {out }}\right)-\operatorname{arsinh}\left(\varphi_{\text {in }}\right)\right),
\end{aligned}
$$

где $\varphi_{\text {in }}=2 \pi R_{\text {in }} / h$ и $\varphi_{\text {out }}=2 \pi R_{\text {out }} / h-$ внутренний и внешний углы нанорулона соответственно.

Нами рассматривается энергия нанорулона, полученного из прямоугольной полоски графена с длиной $L$ и шириной $w$ в направлении оси нанорулона. Энергия $E_{W}$ ван-дер-ваальсового взаимодействия слоев нанорулона пропорциональна площади перекрытия слоев

$$
E_{W}=\frac{\varepsilon w L\left(\varphi_{\text {in }}, \varphi_{\text {out }}-2 \pi\right)}{S_{a}},
$$

где $\varepsilon=52 \mathrm{meV} / \mathrm{atom}-$ энергия взаимодействия слоев графита на один атом углерода [17], $L\left(\varphi_{i n}, \varphi_{\text {out }}-2 \pi\right)$ - длина перекрытия слоев нанорулона, которая равна длине $L$ полоски графена за вычетом длины внешнего слоя, $S_{a}-$ площадь, которая приходится на один атом слоя графена, $S_{a}=3 \sqrt{3} a^{2} / 4=0.0262 \mathrm{~nm}^{2} ; a=0.142 \mathrm{~nm}$ - длина химической связи между атомами углерода в графене. Такой макроскопический подход адекватен для длины перекрытия слоев более $1 \mathrm{~nm}$, т.е. в несколько раз большей, чем характерные расстояния для короткодействующего вандер-ваальсового взаимодействия.

Упругая энергия $E_{a}$ изгиба слоя графена, приходящаяся на один атом, обратно пропорциональна квадрату радиуса $R=h \varphi / 2 \pi$ кривизны слоя $E_{a}=C / R^{2}$, где константа упругости $C=1.79 \mathrm{eV} \cdot \AA^{2} /$ atom. Величина $C$ определена с помощью расчетов методом функционала

Письма в ЖТФ, 2017, том 43, вып. 14 


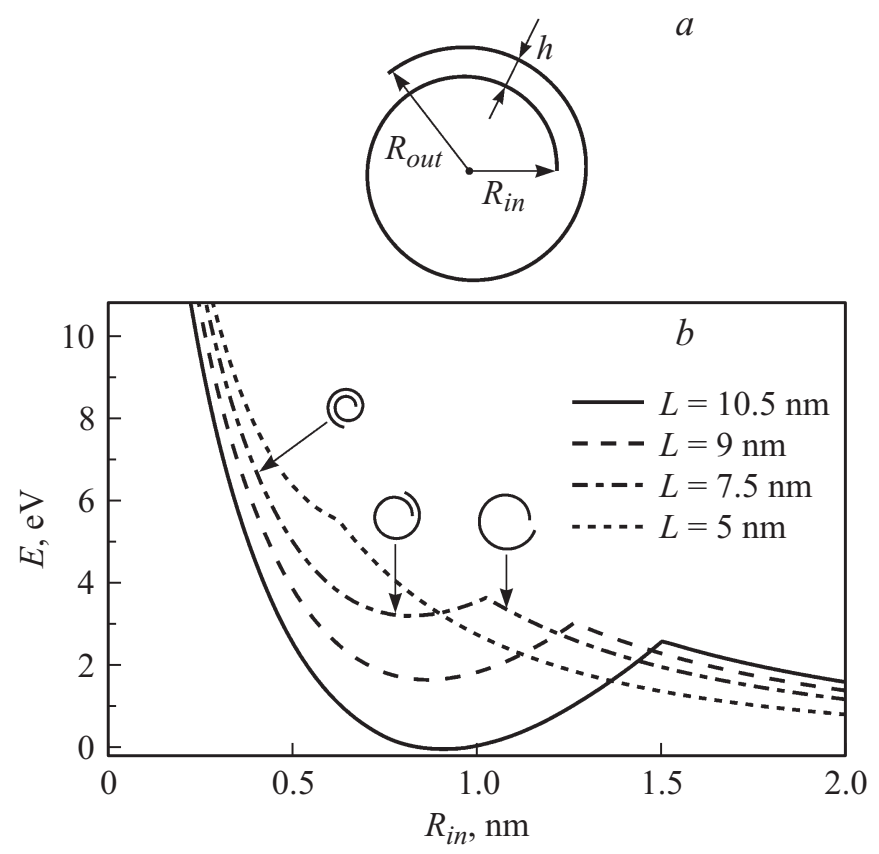

Рис. 1. $a-$ схема нанорулона; ось нанорулона перпендикулярна плоскости рисунка. $b-$ зависимость потенциальной энергии $E$ нанорулона, приходящейся на $1 \mathrm{~nm}$ ширины полоски графена, от внутреннего радиуса $R_{\text {in }}$ для нанорулонов из полоски графена длиной $L=5,7.5,9$ и $10.5 \mathrm{~nm}$.

плотности по зависимости энергии полоски графена от радиуса кривизны [18]. Полную упругую энергию $E_{e l}$ нанорулона получаем аналогично формуле (1) интегрированием $\left(h w E_{a} / 2 \pi S_{a}\right) \sqrt{1+\varphi^{2}}$ по $\varphi$ в виде

$$
\begin{aligned}
& E_{e l}=\frac{2 \pi C w}{h S_{a}} \int_{\varphi_{\text {in }}}^{\varphi_{\text {out }}} \frac{\sqrt{1+\varphi^{2}}}{\varphi^{2}} d \varphi \\
& =\frac{2 \pi C w}{h S_{a}}\left(\frac{\sqrt{1+\varphi_{\text {in }}^{2}}}{\varphi_{\text {in }}}-\frac{\sqrt{1+\varphi_{\text {out }}^{2}}}{\varphi_{\text {out }}}-\operatorname{arsinh}\left(\varphi_{\text {in }}\right)+\operatorname{arsinh}\left(\varphi_{\text {out }}\right)\right)
\end{aligned}
$$


Потенциальная энергия $E$ нанорулона равна $E=E_{e l}-E_{W}$, где $E_{e l}$ и $E_{W}$ даются формулами (3) и (2). Зависимости потенциальной энергии $E$ нанорулона (приходящейся на ширину полоски $w=1 \mathrm{~nm}$ ) от его внутреннего радиуса $R_{\text {in }}$ для различных длин полоски, из которой получен нанорулон, показана на рис. $1, b$. Излом данных зависимостей соответствует внутреннему радиусу $R_{i n}=R_{o v}$, для которого $\varphi_{o u t}-\varphi_{i n}=2 \pi$, и, следовательно, возникает перекрытие слоев нанорулона и появляется отличная от нуля энергия взаимодействия слоев. Минимум на зависимости потенциальной энергии $E$ нанорулона от его внутреннего радиуса $R_{i n}$ соответствует устойчивому состоянию нанорулона. Такой минимум существует только для полосок с длиной $L>L_{m}=6 \mathrm{~nm}$. В рамках используемой макроскопической модели у устойчивого нанорулона из полоски графена с минимально возможной длиной $L_{m}=6 \mathrm{~nm}$ разница внешнего и внутреннего углов равна $\varphi_{\text {out }}-\varphi_{\text {in }}=2 \pi$, а длина перекрытия слоев равна 0 . Длина перекрытия слоев нанорулона в $1 \mathrm{~nm}$ достигается при $L \approx 7 \mathrm{~nm}$. Максимум в зависимости потенциальной энергии $E$ нанорулона от его внутреннего радиуса $R_{\text {in }}$ соответствует потенциальному барьеру $E_{1}$ для сворачивания плоской полоски в нанорулон. Барьер $E_{2}$ для разворачивания нанорулона равен $E_{2}=E_{1}-E_{0}$ и стремится к 0 при стремлении длины $L$ полоски графена при фиксированной ширине $w$ к минимально возможной длине $L_{m}$, для которой возможен устойчивый нанорулон.

Зависимости внутреннего $R_{\text {in }}$ и внешнего $R_{\text {out }}$ радиусов нанорулона в устойчивом состоянии от длины $L$ полоски графена показаны на рис. 2,a. Оба радиуса нанорулона $R_{\text {in }}$ и $R_{\text {out }}$ увеличиваются с увеличением длины $L$ полоски. Зависимость потенциальной энергии $E_{0}$ (приходящейся на ширину полоски $1 \mathrm{~nm}$ ) нанорулона в устойчивом состоянии от длины $L$ полоски графена показана на рис. $2, b$. Для длины полоски $L>10.5 \mathrm{~nm}$ потенциальная энергия $E_{0}$ меньше нуля, т.е. устойчивое состояние нанорулона является основным состоянием полоски графена.

Зависимости барьеров для сворачивания $E_{1}$ и разворачивания $E_{2}$ нанорулона (приходящиеся на ширину полоски $1 \mathrm{~nm}$ ) от длины $L$ полоски графена показаны на рис. $2, b$. Отметим, что величина барьера $E_{1}$ для сворачивания нанорулона уменьшается с увеличением длины $L$ полоски графена. Время жизни $\tau$ нанорулона можно оценить с помощью формулы Аррениуса $\tau^{-1}=\Omega \exp \left(-E_{2} / k T\right)$, где $k-$ постоянная Больцмана, $T$ - температура, $\Omega-$ предэкспоненциальный

Письма в ЖТФ, 2017, том 43, вып. 14 

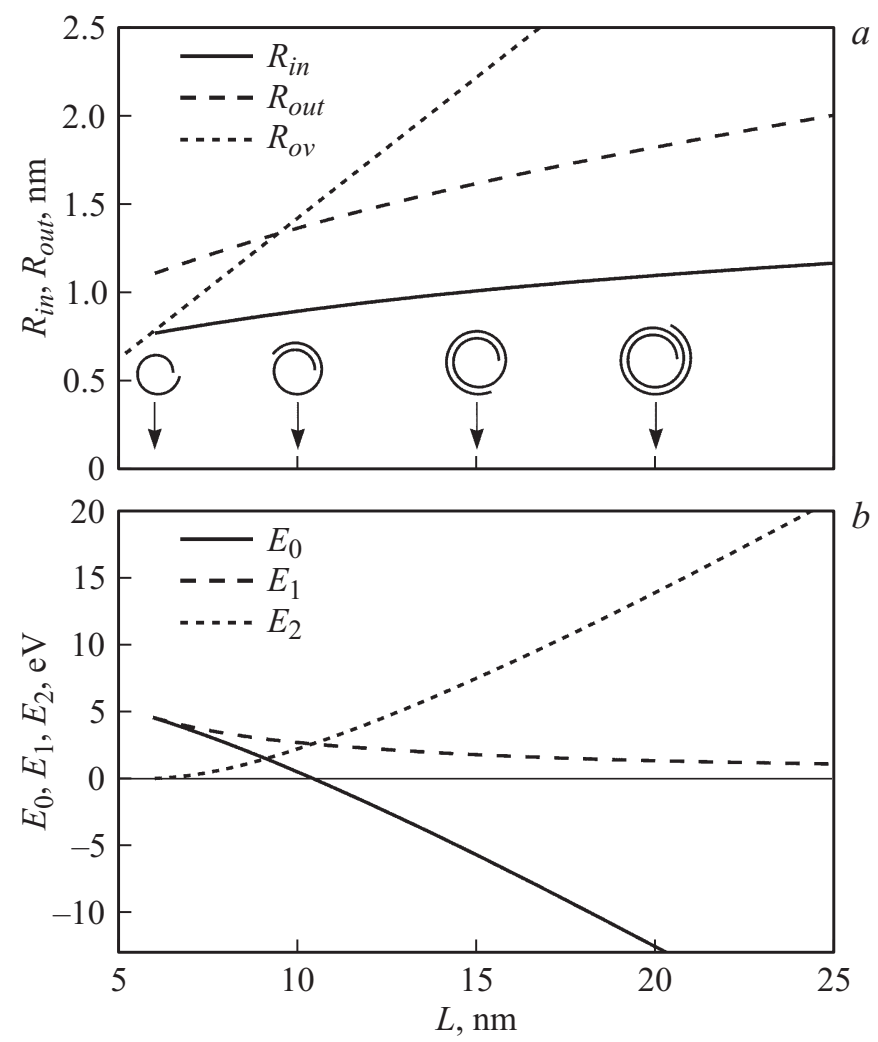

Рис. 2. $a$ - зависимости внутреннего $R_{\text {in }}$ и внешнего $R_{\text {out }}$ радиусов нанорулона в устойчивом состоянии, а также внутреннего радиуса $R_{i n}=R_{o v}$, для которого возникает перекрытие слоев нанорулона от длины $L$ полоски графена, из которой получен нанорулон. $b-$ зависимость потенциальной энергии $E_{0}$ нанорулона в устойчивом состоянии, а также барьеров для сворачивания $E_{1}$ и разворачивания $E_{2}$ нанорулона от длины $L$ полоски графена, из которой получен нанорулон. Приведены значения энергии и барьеров, приходящиеся на $1 \mathrm{~nm}$ ширины полоски графена.

множитель, который по порядку величины равен частоте колебаний, соответствующих разворачиванию нанорулона. Оценим время жизни для нанорулонов с малой длиной перекрытия слоев (по сравнению 


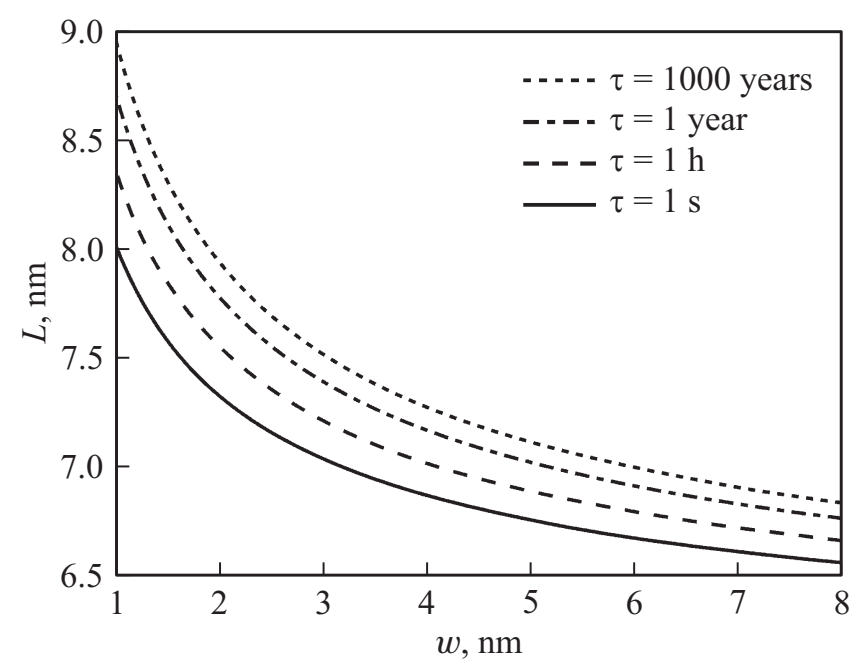

Рис. 3. Размеры (длина $L$ и ширина $w$ ) полоски графена, из которой получен нанорулон, для которых время жизни $\tau$ нанорулона в устойчивом состоянии при комнатной температуре равно $1 \mathrm{~s}, 1 \mathrm{~h}, 1$ year, 1000 years.

с длиной внешнего слоя нанорулона), для которых барьер $E_{2}$ для разворачивания мал, и такие нанорулоны могут разворачиваться в результате термодинамических флуктуаций (см. рис. 3). К разворачиванию нанорулона могут привести колебания, при которых происходит изменение длины перекрытия слоев. Такими колебаниями являются как непосредственно относительные колебания слоев на участке, где слои перекрываются, так и радиальная дыхательная мода, для которой изменение длины перекрытия слоев нанорулона связано с изменением его внутреннего и внешнего радиусов. Частота радиальной дыхательной моды углеродных нанотрубок с радиусом около $1 \mathrm{~nm}-$ около $4 \mathrm{THz}$ [19]. Полагаем, что частота такой моды для нанорулона должна быть меньше, чем для нанотрубки. При малой длине перекрытия слоев можно ожидать, что произойдет небольшое отклонение формы нанорулона от архимедовой спирали, так что относительное положение внутреннего и внешнего слоев нанорулона на участке, где слои перекрываются, будет близко к соизмеримому относительному

Письма в ЖТФ, 2017, том 43, вып. 14 
положению, которое соответствует минимуму энергии взаимодействия слоев [20]. Частота относительных колебаний слоев графена вдоль плоскости слоев в соизмеримом относительном положении не зависит от площади перекрытия слоев и равна $1.5 \mathrm{THz}$ [20], т. е. равна по порядку величины частоте дыхательной моды. В наших оценках используется величина предэкспоненциального множителя $\Omega=1 \mathrm{THz}$. Например, для нанорулона из полоски длиной $L=7.5 \mathrm{~nm}$ и шириной $w=3 \mathrm{~nm}$ барьеры для сворачивания и разворачивания равны $E_{1}=3 \cdot 3.63 \mathrm{eV}$ и $E_{2}=3 \cdot 0.44 \mathrm{eV}$, время жизни $\tau \approx 1.5 \cdot 10^{10} \mathrm{~s} \approx 470$ лет. Рассчитанная зависимость времени жизни нанорулона $\tau$ от длины $L$ и ширины $w$ полоски графена, из которой получен нанорулон, показана на рис. 3 для комнатной температуры. Из рисунка видно, что для устойчивости при комнатной температуре нанорулонов из нанополосок длиной $L>7 \mathrm{~nm}$, для которых длина перекрытия слоев более $1 \mathrm{~nm}$, достаточно размера нанорулона в направлении его оси $w$ менее $10 \mathrm{~nm}$. Это позволит использовать такие нанорулоны в наноэлектромеханических системах и для разработки новых наноматериалов.

Таким образом, расчетным способом исследованы структура, размеры, энергетические характеристики для сворачивания и разворачивания нанорулона из прямоугольной полоски графена, а также определено его время жизни в устойчивом состоянии.

Работа выполнена при финансовой поддержке Российского фонда фундаментальных исследований (проект 16-52-00181-Бел) и Белорусского республиканского фонда фундаментальных исследований (проект Ф16P-107).

\section{Список литературы}

[1] Viculis L.M., Mack J.J., Kaner R.B. // Science. 2003. V. 299. P. 1361.

[2] Savoskin M.V., Mochalin V.N., Yaroshenko A.P. et al. // Carbon. 2007. V. 45. P. 2797-2800.

[3] Roy D., Angeles-Tactay E., Brown R.J.C. et al. // Chem. Phys. Lett. 2008. V. 465. P. 254-257.

[4] Xie X., Ju L., Feng X. et al. // Nano Lett. 2009. V. 9. P. 2565-2570.

[5] Zheng B., Xu Z., Gao C. // Nanoscale. 2016. V. 8. P. 1413-1420.

[6] Pan H., Feng Y., Lin J. // Phys. Rev. B. 2005. V. 72. P. 085415.

[7] Shi X., Pugno N.M., Gao H. // J. Comput. Theor. Nanosci. 2010. V. 7. P. 517521. 
[8] Shi X., Pugno N.M., Gao H. // Int. J. Fracture. 2011. V. 171. P. 163-168.

[9] Savin A.V., Korznikova E.A., Dmitriev S.V.// Phys. Rev. B. 2015. V. 92 P. 035412.

[10] Shi J., Yin H., Yu J. et al. // Comput. Mater. Sci. 2016. V. 125. P. 146-153.

[11] Zeng F., Kuang Y., Liu G. et al. // Nanoscale. 2012. V. 4. P. 3997-4001.

[12] Braga S.F., Coluci V.R., Baughman R.H., Galvão D.S. // Chem. Phys. Lett. 2007. V. 441. P. 78-82.

[13] Coluci V.R., Braga S.F., Baughman R.H., Galvão D.S. // Phys. Rev. B. 2007. V. 75. P. 125404.

[14] Mpourmpakis G., Tylianakis E., Froudakis G.E. // Nano Lett. 2007. V. 7. P. $1893-1897$.

[15] Khaledian M., Ismail R., Saeidmanesh M., Khaledian P. // RSC Adv. 2015. V. 5. P. 54700-54709.

[16] Воднев В.Т., Наумович А.Ф., Наумович Н.Ф. Основные математические формулы. Минск: Вышэйшая школа, 1988.

[17] Zacharia R., Ulbricht H., Hertel T. // Phys. Rev. B. 2004. V. 69. P. 155406.

[18] Lebedeva I.V., Popov A.M., Knizhnik A.A. et al. // Nanoscale. 2012. V. 4. P. 4522 4529.

[19] Souza Filho A.G., Chou S.G., Samsonidze Ge.G. et al. // Phys. Rev. B. 2004. V. 69. P. 115428.

[20] Lebedeva I.V., Knizhnik A.A., Popov A.M. et al. // Phys. Chem. Chem. Phys. 2011. V. 13. P. 5687-5695.

Письма в ЖТФ, 2017, том 43, вып. 14 